\title{
EFEITO DE DESFOLHANTES NA CULTURA ALGODOEIRA (Gossypium hirsutum L.) NO TRIÂNGULO MINEIRO
}

\section{RESUMO}

Foram testados 4 desfolhantes com diferen tes doses, e em misturas, sobre a cultivar IAC 13-1 em solo argiloso de Ipiaçú-MG. Os tratamentos constituiram de 0,71 e $1,42 \mathrm{~kg}$ i.a./ha de folex (71,2\% de fosforotritoato de tributila), 1,06 e 1,41 $\mathrm{kg}$ i.a./ha de def $(70,5 \%$ de s,s,s-tributiltritiofosfato), 0,36 e $0,54 \mathrm{~kg}$ i.a.fha de paraquat $(36,2 \%$ de 1,1 dimetil-4,4' bipiridilio-dicloreto, 1,92 $\mathrm{kg}$ i.a./ha de etileno $(48 \%$ de ácido cloroetilfosfórico), paraquat $+\operatorname{def}(0,36+0,70 \mathrm{~kg}$ i.a./ha), paraquat + def. $(0,54+1,41 \mathrm{~kg}$ i.a./ha), paraquat + folex $(0,36+0,70 \mathrm{~kg}$ i.a. $/$ ha $)$, paraquat + folex $(0,54+1,42 \mathrm{~kg}$ i.a. $/ \mathrm{ha})$, paraquat $(0,36 \mathrm{e}$ $0,54 \mathrm{~kg}$ i.a./ha) e urna testemunha isenta de desfolhantes.

As aplicações foram feitas com $70 \%$ a $80 \%$ de capulhos abertos usando 8001 de água/ha. A avaliação dos resultados consistiu na contagem de folhas secas + verdes e dos efeitos dos desfolhantes sobre plantas daninhas segundo a escala E.W.R.C. Def $(1,41 \mathrm{~kg}$ i.a./ha), paraquat + folex $(0,54+1,42$ kg i.a./ha) e paraquat $+\operatorname{def}(0,54+1,41 \mathrm{~kg}$ i.a./ha) foram os melhores na avaliação feita 5 dias após a aplicação. O rebrotamento, causado pelas chuvas, igualou todos os tratamentos aos 10 dias, diferenciando-os apenas da testemunha e, aos 15 dias, igualando-os a ela. Todos os tratamentos com paraquat resultaram em melhor controle sobre plantas daninhas que os demais, contribuindo para facilitar a colheita mecânica e melhorar o tipo de algodão. Os tratamentos estudados não afetaram as características tecnológicas da fibra.

UNTTERMOS: desfolhantes, herbicidas, algodão, plantas daninhas.

\section{SUMMARY}

EFFECTS OF DEFOLIANTS ON COTTON (Gossypium hirsutum L.) AT TRIÂNGULO MINEIRO.

\section{J.P. Del C. LACA-BUENDIA* \& L. FERREIRA**}

* Eng..$^{\circ}$ Agr. ${ }^{\circ}$ - Coordenador do Projeto Algodão EPAMIG. Caixa Postal 515 - Belo Horizonte, MG $-30.000$

** Eng. ${ }^{\circ}$ Agr. $^{\circ}$ - Pesquisador da EPAMIG, Caixa Postal 351 - Uberaba, MG - 38.100.

Trabalho apresentado no XI Seminário Brasi leiro de Herbicidas e Ervas Daninhas. IAPAR, Londrina, PR. 20 a 22 julho/76.

Recebido para publicação em 10.10.79.

Four defoliants were tested, alone and blended at different rates on cotton cv. IAC 13-1 on clay soil of Ipiaçu-MG. The treatments consisted of 0,71 and $1,42 \mathrm{~kg}$ i.a./ha of folex $(71,2 \%$ of tributyl phosphorotrithioate), 1,06 and $1,41 \mathrm{~kg}$ i.a./ha of def $(70,5 \%$ of $\mathrm{s}, \mathrm{s}, \mathrm{s}, \quad$ - tributyl phosphorotrithioate), 0,36 and $0,54 \mathrm{~kg}$ i.a./ha of paraquat $\left(36,2 \%\right.$ of $1^{\prime} 1$ dimethyl-4-4' bipyridyldiylium ion), $1,92 \mathrm{~kg}$ i.a./ha of ethrel (48\% of chlorethyphosphoric acid), paraquat + def $(0,36+0,70 \mathrm{~kg}$ i.a. /ha), paraquat + folex $(0,36+$ $0,70 \mathrm{~kg} \mathrm{La} / \mathrm{ha})$, paraquat + folex $(0,54+1,42 \mathrm{~kg}$ i.a./ha), paraquat $+\operatorname{def}(0,54+1,41 \mathrm{~kg}$ i.a./ha), paraquat $(0,36$ and $0,54 \mathrm{~kg}$ i.a./ha) and a check. Each treatment was repeated four times. Spray were done when $70 \%$ to $80 \%$ bolls were open using 8001 of water/ha. Data were taken from the number of dried + green leaves and the effect of defoliants weeds by the E.W.R.C. scale. Def $(1,41 \mathrm{~kg}$ i.a./ha), paraquat + folex $(0,54+1,42 \mathrm{~kg}$ i.a./ha) and paraquat $+\operatorname{def}(0,54+1,41 \mathrm{~kg}$ i.a. $/$ ha) were the best treatments 5 days after spraying. New growth induced by rains equalized all treatments, except the check, 10 days after the application and including the 5 days later. Paraquat alone or blended, was the best product to control weeds making easier the mechanical harvester work and improving the raw-cotton quality.

KEYWORDS: Defoliants, herbicides, cotton, weeds.

\section{INTRODUÇÃO}

Com a colheita mecanizada, surgiu a necessidade do uso de desfolhan tes na cultura algodoeira. A primeira desfolha com sucesso, foi efetuada por Hall \& Harrel, utilizando cianamida de cálcio em pó, para o controle de doenças fúngicas das maçãs do algodoeiro 
(5). Kramer \& Farah (6), estudando doses de mata-ervas tipo A, à base de clorato de sódio, obtiveram $80-90 \%$ de abcisão de folhas 10 dias após a aplicação e 95\% após $30^{\circ}$ dia, aplicando $8,0 \mathrm{~kg}$ do desfolhante/ha. Vargas \& Bravo (8), testando 4 desfolhantes comerciais, observaram que alguns produtos causaram aumento de produção na primeira colheita, e que altas concentrações não derrubavam bem as folhas, provavelmente devido à morte prematura da planta, sem terminar o processo de abcisão. Brown \& Rhyne (1) concluiram que o estágio de maturação das maçãs tinha influência na queda das folhas. Também os solos mais férteis, mais úmidos e plantas muito frutificadas ajudam na desfolha química. Bons resultados foram conseguidos com o uso de def, folex, e paraquat puros ou em misturas, dentre vários desfolhantes estudados $(2$, 3, 4 e 7 ).

O objetivo deste trabalho foi determinar o desfolhante mais eficiente e a dose mais econômica para o algodoeiro na Região do Triângulo Mineiro. Verificou-se ainda a influência nos rendimentos e na qualidade da fibra.

\section{MATERIAIS E MÉTODOS}

Este ensaio foi instalado em Ipiaçu-MG, em solo argiloso, em cultura da cultivar IAC 13-1, já estabelecida no campo. O delineamento experimental foi de blocos casualizados, com 12 tratamentos e 4 repetições. As parcelas tinham 4 fileiras de $10 \mathrm{~m}$ de comprimento e espacadas de $1 \mathrm{~m}$, ocupando uma área de $40 \mathrm{~m}^{2}$. As duas fileiras centrais formavam a área útil de $20 \mathrm{~m}^{2}$.

Os tratamentos foram os seguintes:

- folex - concentrado emulsionável com $71,2 \%$ de fosforotritoato de tributila $(0,71$ e $1,4 \mathrm{~kg}$ i.a./ha);

- def - concentrado emulsionável, com $70,5 \%$ de s,s,s-tributil-tritiofosfato (1,06 e 1,41 kg i.a./ha);

- paraquat - concentrado emulsionável, com $36,2 \%$ de $1,1^{\prime}$ dimetil - 4,4' - bipiridilio-dicloreto $(0,36$ e $0,54 \mathrm{~kg}$ i.a./ha);

- $\quad$ ethrel - concentrado emulsionável, com 48\% de ácido cloroetilfosfórico (1,92 kg i.a./ha);

- $\quad$ paraquat $+\operatorname{def}(0,36+0,70 \mathrm{~kg}$ i.a. $/ \mathrm{ha})$

- $\quad$ paraquat $+\operatorname{def}(0,54+1,41 \mathrm{~kg}$ i.a. $/ \mathrm{ha})$;

- $\quad$ paraquat + folex $(0,36+0,70 \mathrm{~kg}$ i.a. $/ \mathrm{ha})$;

- paraquat + folex $(0,54+1,42 \mathrm{~kg}$ i.a. $/ \mathrm{ha})$;

- paraquat $(0,36$ e $0,54 \mathrm{~kg}$ i.a. $/$ ha) e uma testemunha, sem aplicação de nenhum produto.

As aplicacões foram feitas com $70 \%$ e $80 \%$ de maçãs abertas (29.03.75) com um pulverizador costal motorizado dotado de bico D-2, com gasto de 8001 de água por hectare.
Os dados foram tomados através de 6 plantas marcadas nas duas linhas centrais de cada parcela. Registrou-se o número de folhas verdes + secas, antes da aplicação dos desfolhantes e 5, 10 e 15 dias após a aplicação. Junto à contagem de 10 dias após a aplicação, foi feita uma avaliação do efeito herbicida de cada tratamento sobre as plantas daninhas existentes nas parcelas, usando a escala E.W.R.C.

$\mathrm{Na}$ primeira colheita feita manualmente 20 dias após a aplicação foram tomadas da área útil de cada parcela, 20 capulhos do terço superior das plantas, marcadas antes da aplicação dos desfolhantes para análise dos componentes de produção e qualidade da fibra.

\section{RESULTADOS E DISCUSSÃO}

Não houve significância estatística entre os dados de "stand" e altura das plantas, tomadas antes das aplicações e na colheita, bem como, para rendimento, peso de capulho e de cem sementes, percentagem, indice e características tecnológicas da fibra (quadro 1). A análise de variância para a contagem do número de folhas, feita antes da aplicação, mostrou ser uniforme a relação folhas/planta em todo o ensaio (quadro 2). Cinco dias após a aplicação, verificou-se que o número de folhas verdes + secas mostrava diferença significativa entre os tratamentos estudados e a testemunha, sendo que def $(1,41 \mathrm{~kg}$ i.a./ha), paraquat + folex $(0,54+1,42 \mathrm{~kg}$ i.a./ha) e paraquat $+\operatorname{def}(0,54+1,41 \mathrm{~kg}$ i.a./ha), também se diferenciavam estatisticamente do tratamento com o ethrel $1,92 \mathrm{~kg}$ i.a./ha, sem contudo diferirem dos demais. Após 5 dias, houve rebrotamento o que fez com que nas contagens realizadas 10 e 15 dias após a aplicação, os tratamentos se igualassem, diferenciando significativamente da testemunha com 10 dias e igualando-se a ela 15 dias após a aplicação (Quadro 2 e Figura 1).

Estes dados não concordam com os obtidos por Cruz \& Leiderman (3) que indicam mais de $80 \%$ de desfolha após 20 dias da aplicação com a mistura de def + paraquat. Por outro lado, estão de acordo com Burhan \& Gleadle (2), que mostrou desfolha maior para folex e def entre 4 e 11 dias após a aplicação. 
Quadro 1 - Resultados médios obtidos no ensaio do efeito de desfolhantes na cultura algodoeira (G. hirsutum L.) em Ipiaçú - Triângulo Mineiro, no ano agricola de 1974/75.

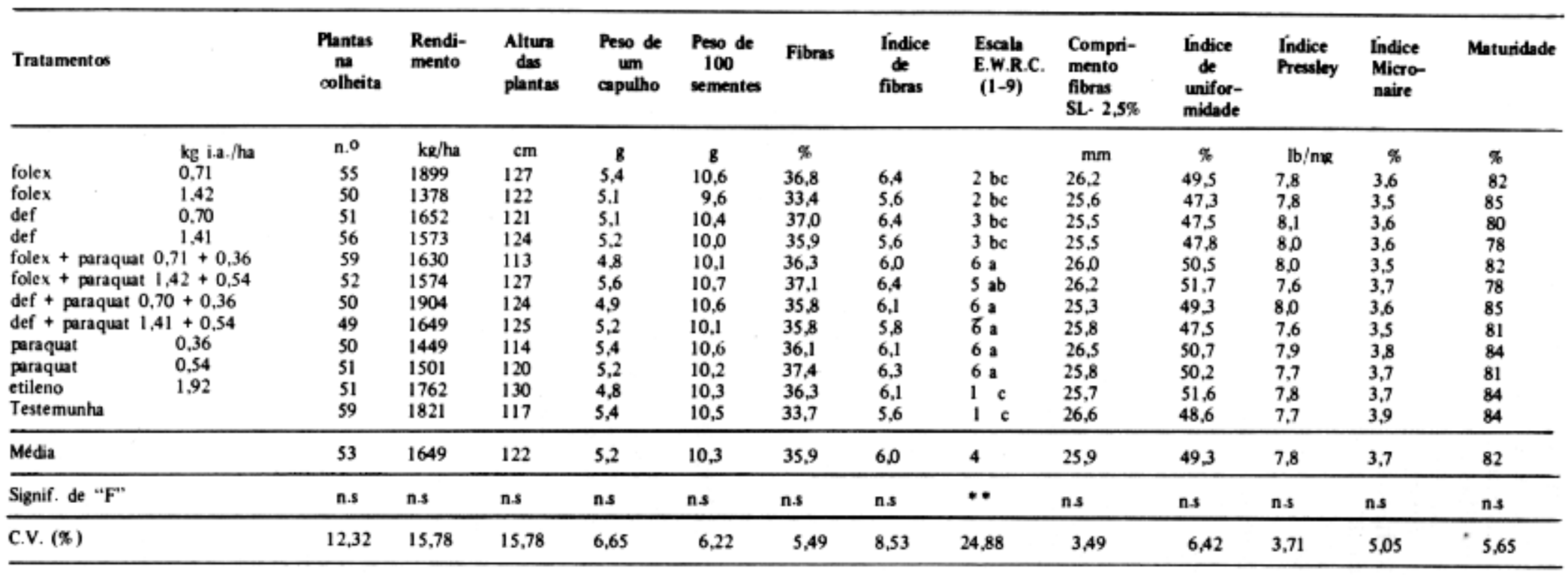

OBS.: As médias na mesma coluna assinaladas pela mesma letra, nåo diferem significativamente entre si, ao nivel de $5 \%$ pelo teste de Tukey. 
Quadro 2 - Resultados do número de folhas e percentagem de queda na aplicação de desfolhantes na cultura algodoeira (G. hirsutum $L$.), em Ipi açú - Triângulo Mineiro, no ano agrícola de 1974/75.

\begin{tabular}{|c|c|c|c|c|c|c|c|c|}
\hline \multirow{2}{*}{\multicolumn{2}{|c|}{ TRATAMENTOS }} & \multicolumn{4}{|c|}{ Número de folhas por planta } & \multicolumn{3}{|c|}{ Percentagem de queda, em relação ao n.0 inicial } \\
\hline & & $\begin{array}{l}\text { Antes da } \\
\text { Aplicaço }\end{array}$ & 5 dins após & 10 dias após & 15 dias após & 5 dias após & 10 dias após & 15 dias após \\
\hline $\begin{array}{l}\text { folex } \\
\text { folex } \\
\text { def } \\
\text { def } \\
\text { paraquat }+ \\
\text { paraquat + } \\
\text { paraquat + } \\
\text { paraquat }+ \\
\text { paraquat } \\
\text { paraquat } \\
\text { etileno } \\
\text { Testemunha }\end{array}$ & $\begin{array}{c}\mathbf{k g ~ i . a . / h a} \\
0,71 \\
1,42 \\
0,70 \\
1,41 \\
+0,71 \\
+1,42 \\
0,70 \\
1,41 \\
0,36 \\
0,54 \\
1,92\end{array}$ & $\begin{array}{l}38,2 \\
43,2 \\
38,9 \\
29,9 \\
32,0 \\
42,0 \\
34,0 \\
33,9 \\
35,7 \\
38,5 \\
42,9 \\
38,0 \\
\end{array}$ & $\begin{array}{l}3,9 \mathrm{ab} \\
3,2 \mathrm{ab} \\
4,9 \mathrm{ab} \\
1,8 \mathrm{a} \\
3,2 \mathrm{ab} \\
2,1 \mathrm{a} \\
2,8 \mathrm{ab} \\
1,8 \mathrm{a} \\
3,2 \mathrm{ab} \\
3,7 \mathrm{ab} \\
10,0 \mathrm{~b} \\
24,0 \mathrm{c}\end{array}$ & $\begin{array}{r}8,8 \mathrm{a} \\
9,3 \mathrm{a} \\
10,3 \mathrm{a} \\
7,1 \mathrm{a} \\
7,4 \mathrm{a} \\
5,5 \mathrm{a} \\
6,7 \mathrm{a} \\
7,2 \mathrm{a} \\
8,3 \mathrm{a} \\
8,1 \mathrm{a} \\
18,2 \mathrm{ab} \\
24,0 \mathrm{c}\end{array}$ & $\begin{array}{l}23,4 \text { a } \\
22,6 \text { a } \\
21,0 \text { a } \\
21,0 \text { a } \\
19,8 \text { a } \\
16,1 \text { a } \\
19,1 \text { a } \\
19,8 \text { a } \\
19,5 \text { a } \\
17,9 \text { a } \\
41,3 \text { a } \\
41,2 \text { a }\end{array}$ & $\begin{array}{l}89,79 \\
92,59 \\
87,40 \\
93,98 \\
90,00 \\
95,00 \\
91,76 \\
94,69 \\
91,04 \\
90,39 \\
76,69 \\
36,84\end{array}$ & $\begin{array}{l}76,96 \\
78,47 \\
73,52 \\
76,25 \\
76,87 \\
86,90 \\
80,29 \\
78,76 \\
76,75 \\
78,96 \\
57,57 \\
36,84\end{array}$ & \begin{tabular}{r|}
38,74 \\
47,68 \\
46,01 \\
29,76 \\
38,12 \\
61,67 \\
43,82 \\
41,59 \\
45,38 \\
53,51 \\
3,73 \\
8,42
\end{tabular} \\
\hline Média & & 36,8 & 5,4 & 10,4 & 23,6 & & & \\
\hline Valor de " $F$ & & n.s. & $* *$ & $* *$ & * & & & \\
\hline C.V. $(\%)$ & & 28,71 & 47,64 & 49,65 & 46,71 & & & \\
\hline
\end{tabular}

OBS.: As médias na mesma coluna, assinaladas pela mesma letra, não diferem significativamente entre si, ao nível de $5 \%$, pelo teste de Tukey. 


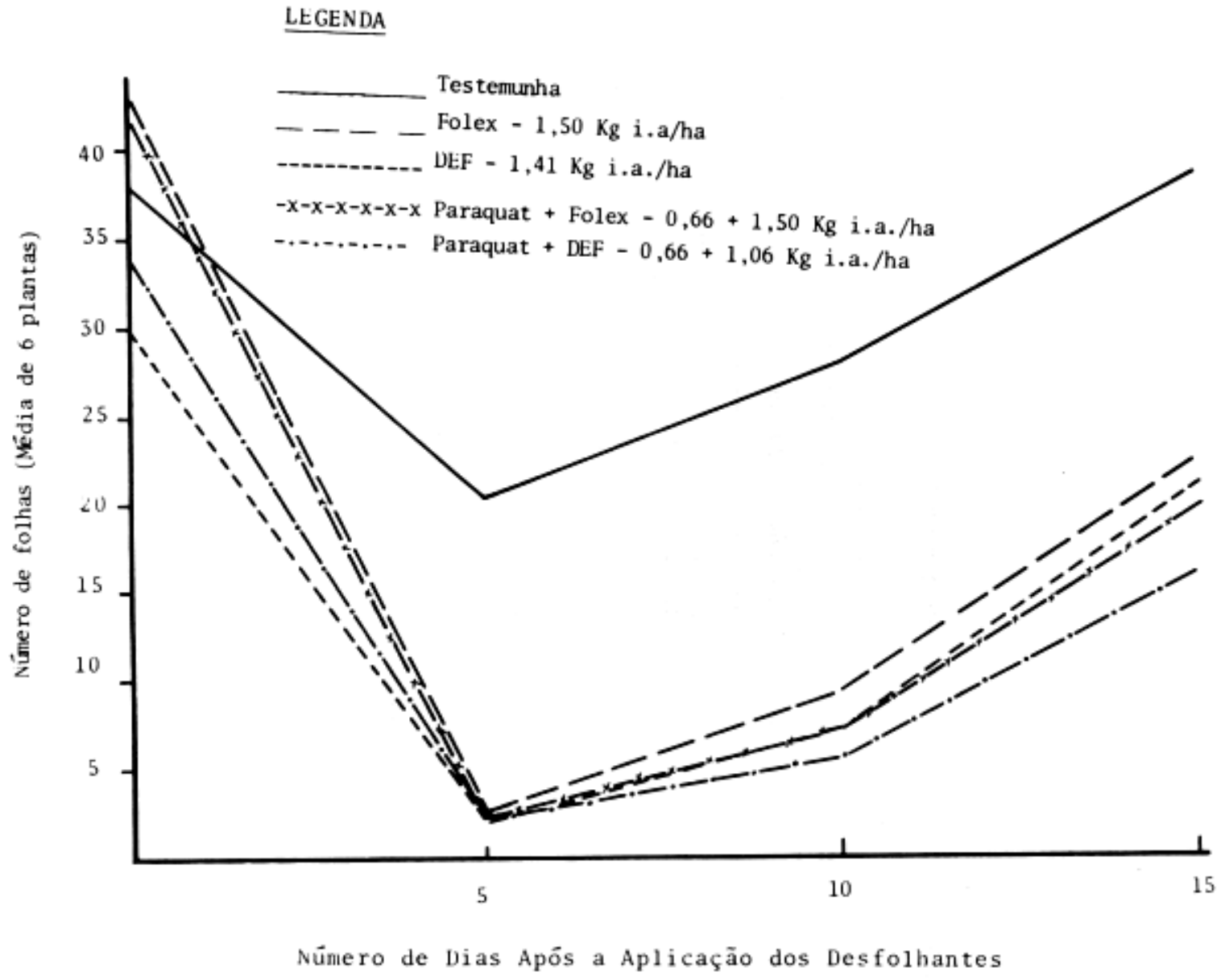

Figura 1 - Efeito da aplicação de desfolhantes em algodáo (Gossypium hirsutum L.) na queda de folhas. Triàngulo Mineiro, ano agrícola 1974/75.

Os tratamentos que continham paraquat apresentaram melhor controle sobre as plantas daninhas presentes por ocasião da colheita. Este controle facilita a colheita e melhora o tipo do algodão (Quadro 1).

\section{LITERATURA CITADA}

1. Brown, L.C. \& Rhyne, C.L. Chemical defoliation of cotton. II - The influence of boll maturity on the desfoliability of species and varieties of cotton. Agronomy Journal, 46(3): 132, 1954.

2. Burhan, H.O. \& Gleadle, G.E. Trials of chemical defolation of cotton in the Sudan. Cotton Growing Rev. 50(40): 327-83, 1973.

3. Cruz, L.S.P. \& Leiderman, L. Efeitos de compostos Bipiridilium e suas misturas empregadas como desfolhantes para o algodoeiro. In Seminário Brasileiro de Herbicidas e Ervas
Daninhas, $10 .^{\circ}$, Sta. Maria, R.S., 1974. Resumos p.36.

4. Cruz, L.S.P. \& Leiderman, L. Trakephon. Um novo desfolhante para algodoeiro. In: Seminario Brasileiro de Herbicidas e Ervas Daninhas, $100^{\circ}$, Sta. Maria, R.S., 1974. Resumos, p.30.

5. Eaton, F.M. Physiology of the cotton plant. Annual review of plant physiology, p.229-328, 1955.

6. Kramer, M. \& Farah, J.B. Nota prévia sobre um desfolhante para algodāo, à base de clorato de sodio. In: Anais do Seminário Brasileiro de Herbicidas e Ervas Daninhas, $1^{\circ}$, Rio de Janeiro, RJ., 1955. p.197-200.

7. Santos, C.A.L. dos, Leiderman, L. \& Grassi, N. Aplicaçóes de desfolhantes na cultura do algodáo. O Biológico 36: 147-151, 1970.

8. Vargas, R.S. \& Bravo, M.C. Ensayo preliminar com três desfoliantes el algodonero. Lima, Ministério da Agricultura (SIPA), 6p. (Informe n. ${ }^{\circ}$ 26). Abril, 1964 . 